\title{
Translation and Validation of Patient Activation Measure (PAM®-13) in Kannada Language
}

\author{
Angela Arun Kapoor ${ }^{1}$, Prem Venkatesan², Pratik Arun Phansopkar³ \\ ${ }^{1}$ Department of Cardiorespiratory Physiotherapy, Ravi Nair Physiotherapy College, Datta Meghe Institute of \\ Medical Sciences, Wardha, Maharashtra, India. ${ }^{2}$ Department of Physiotherapy, School of Allied Health \\ Sciences, Manipal University, Bangalore, Karnataka, India. ${ }^{3}$ Department of Musculoskeletal Physiotherapy, \\ Ravi Nair Physiotherapy College, Datta Meghe Institute of Medical Sciences, Wardha, Maharashtra, India.
}

\section{ABSTRACT}

\section{BACKGROUND}

In a country like India with a large population, with diverse culture, socioeconomic status and educational levels, there is a need to translate a Patient Activation Scale Measure (PAM®13) into a regional language which will reduce the language barrier, increases the understanding of patient's disease condition, and improves their self-management skills. Hence, a reliable and validated instrument 'Patient Activation Measure (PAM $® 13)$ ' is used for evaluating patient's awareness, skills, and trust in self-management of disease. We wanted to translate and validate the PAM $®-13$ questionnaire in Kannada language and assess activation levels in diabetes mellitus patients.

\section{METHODS}

A cross-sectional analysis done among 200 adults with diabetes mellitus who speak Kannada. Patients of diabetes mellitus, aged 18 - 85 years from both sexes were included in the study. The PAM®-13 - was translated as recommended by World Health Organisation's procedure for cross-cultural validation and adaptation of selfreport measures. This involved forward translation, synthesis, backward translation, pre-testing and the final version which was performed by the professionals of Kannada and English language. Data was analysed using SPSS ver. 24.0 for mean, median, standard deviation, Pearson's correlation and Spearman's correlation. Stata 14 was used to analyse internal consistency using Cronbach's Alpha, inter-item, inter-rest, inter-test correlation.

\section{RESULTS}

We found that the mean of the Kannada version of the PAM $\AA-13$ was 55.68. The level of internal consistency was good $(\alpha=0.8357)$.

\section{CONCLUSIONS}

PAM ${ }^{\circledR}-13$ in Kannada language has been demonstrated to be a valid and reliable measure of patient activation in the diabetic population and the present study suggests good psychometric properties.

\section{KEY WORDS}

Patient Activation, Self-Management, Diabetes, Chronic Illness, Chronic Disease, PAM $® 13$, Psychometric Properties
Corresponding Author: Dr. Pratik Arun Phansopkar. Assistant Professor,

Department of Musculoskeletal Physiotherapy, Ravi Nair Physiotherapy College, Datta Meghe Institute of Medical Sciences, Wardha, Maharashtra, India. E-mail:drpratik77@gmail.com

\section{DOI: $10.14260 / j e m d s / 2020 / 653$}

\section{How to Cite This Article:}

Kapoor AA, Venkatesan P, Phansopkar PA. Translation and validation of patient activation measure (PAM®-13) in Kannada language. J Evolution Med Dent Sci 2020;9(40):2981-2986, DOI: $10.14260 / \mathrm{jemds} / 2020 / 653$

Submission 04-07-2020,

Peer Review 28-08-2020,

Acceptance 04-09-2020,

Published 05-10-2020.

Copyright (c) 2020 Angela Arun Kapoor. This is an open access article distributed under Creative Commons Attribution License [Attribution 4.0 International (CC BY 4.0)] 


\section{BACKGROUND}

Chronic illness is of growing concern, especially in primary health care centres. ${ }^{1}$ Promoting active patient participation in managing their illness is an important task for the general practitioner. ${ }^{2}$ The Chronic Care Model was established for the management of chronic care and practice improvement which delivers help to healthcare providers to improve patient's health outcomes 3,4 and cost savings. ${ }^{5}$ The cornerstone of chronic care model is collaborative care and patient activation which seeks to empower patients in their care and support self-management. ${ }^{6}$

To manage a chronic illness, patients need to achieve their knowledge, skills, beliefs and behaviours about one's health which are integral parts in making a patient activated. ${ }^{7}$ Evidence shows that these patient activation measures can lead to better self-management in patients with chronic conditions such as angina, chronic pain, depression, diabetes and hypertension.8,9,10 India, the burden continues to increase during next 25 years as a consequence of rapidly ageing population of India. In 2004, mortality rate in India was 10.3 million with 5.2 million (50\%) due to age specific chronic diseases with 769 per 100000 men (56\%) and 602 per 100 000 women $(100 \%)$ was estimated to be higher in India than in high-income countries. ${ }^{11}$

Amongst these chronic conditions, Diabetes is one of the most prevailing conditions and a major cause of morbidity, disability and mortality. Many valid and reliable outcome measures have been used to assess different aspects of activation in the clinical research settings. ${ }^{7}$ Nonetheless, these measures tend to focus on a single behaviour. In 2005, a modified version of the Patient Activation Measure (PAM®13) was developed by Judith Hibbard and colleagues. This questionnaire focuses on four elements i.e. knowledge, skills, confidence, behaviour with chronic illness, is a 13-item short form which suggests four levels of activation that patients reach by becoming fully engaged in managing their own health. The PAM $®-13$ was considered as a reliable and valid questionnaire. 8

The validity of PAM ${ }^{\circledR}-13$ has been proven as a measure of the level of activation in patients with chronic illness in United States. ${ }^{8}$ To our knowledge, the result of the research states that the validity of PAM $@-13$ has been conducted in languages such as Italian, German, Hebrew, Danish, Netherlands and but not in the non-English speaking countries including the Asian countries. ${ }^{12}$

The level of patient activation depends on knowledge about their health condition, availability of treatment options, self-care as well as cultural values. Therefore, it is important to consider the cultural differences that exist in various regions. In a country like India with large population, presence of cultural heterogeneity, difference in socioeconomic status and educational levels, there is a need to translate a patient activation scale measure (PAM®13) into a regional language which will reduce the language barrier, increase understanding of patient's disease condition and will improve their self-management skills.

Hence, this research was an aim to translate and validate PAM $®-13$ in one of the Regional languages - Kannada and validate in patients with diabetes mellitus considering the high prevalence of the disease in southern states of India.

\section{METHODS}

In Manipal Hospital, Bangalore, a cross sectional study was conducted. In this study, we included 200 adults with diabetes mellitus who speak Kannada. The study gathered demographic data about age, gender, disease severity and educational level. Patients aged between 18 and 85 years of both male and female with diabetes mellitus that were able to read, understand and write Kannada language.

The study has been approved by the Committee on Institutional Review. Upon receiving permission from Insignia Health Inc., the PAM $®-13$ was translated as suggested by the protocol for cross-cultural validation and self-reporting steps of the World Health Organization for cross-cultural validation and adaptation of self- report measures. The department of Kannada and English Language Bangalore University was then approached for the process of translation. ${ }^{13}$ This involves forward translation, synthesis, backward translation, pre-testing and the final version which was performed by the professionals of Kannada and English language.

\section{Translation and Cultural Adaptation of PAM $₫-13$}

The method of translation included the following steps:

\section{Forward Translation (T)}

Two forward translations were made of the questionnaire from the English language to the Kannada language by the translator whose mother tongue was Kannada language and also knew the English language who produced the two independent translations.

Translator 1 (T1) - Translator 1 was informed about the concepts being examined, which lead to a more clinical outlook and a reliable equivalence of the questionnaire being translated.

Translator 2 (T2) - Translator 2 was uninformed about the concepts being quantified and with no medical background. This translation will reflect the language used by that population, it will be less impactful and be unclear than the original questionnaire.

\section{Synthesis (T-12)}

The translation was compared and discrepancies in the construction of sentences and words were noted and resolved in a discussion between the translators. The two translators and a third translator together synthesised the translation i.e., work done by a 1st translator (T1) and 2nd Translator (T2) producing one common translation (T-12), with a written report carefully documenting the synthesis process and resolving the addressed issues.

\section{Backward Translation (BT)}

The final forward translated version (T-12) was back translated from the Kannada Language into original language of the questionnaire i.e. English Language. 
The two independent translators with the English language as their mother tongue translated T-12 into BT-1 and BT-2.To avoid information bias and to draw unexpected meanings of the items in the translated questionnaire (T-12), the two translators were kept blinded to the original version, unformed of the concepts of questionnaire an $\mathrm{d}$ were without a medical background. A translator then translated the questionnaire back into the English language. This translated version reflects the same item content as the original version and intends to capture the purpose of it. The emphasis of back translation was on conceptual and cultural equivalence and not linguistic equivalence.

\section{4. $\quad$ Pretesting}

Pre - testing of the questionnaire was done on 10 patients with diabetes mellitus. Each subject completed the questionnaire and was interviewed to probe about what the subjects thought was meant by each questionnaire item and chosen responses. Both the meaning of the items and responses were noted. This ensured that the adapted version was still retaining its equivalence in the applied situations. The answers to the questions were compared to the actual response to the questionnaire for consistency.

\section{Final Version}

The final version of PAM®-13 in the Kannada language was prepared for its applicability. The purpose of the study and its intent was explained in detail to all the subjects of the legally acceptable representative in their native language and a written informed consent was obtained as per the protocol. Subjects were enrolled after screening according to the inclusion and exclusion criteria. Demographic details of subjects were taken at baseline. The patient activation was assessed in Bangalore population post translation. According to Insignia Health Inc. guidelines, the raw scores were transformed through natural logarithm to achieve a better expression of the relative distance between the scores. The items were transformed to a standardized metric ranging from $0-100(0=$ lower activation; 100 higher activations), to compare Kannada results to the original data. The score was calculated by summing up the raw scores and mapping up the sum onto a scale of 0-100. A higher score of PAM-13 indicates a higher level of activation.

\section{Patient Activation Measures}

After securing permission from Insignia Health Inc. the PAM $\AA-13$ - was translated as recommended by World Health Organisation's procedure for cross-cultural validation and adaptation of self- report measures.

The importance of patient activation mandates the need for robust tools to measure and improve care for people with chronic conditions. In 2004, Judith Hibbard and colleagues developed patient activation measure originally as a 22 item Scale, (PAM $®-22)$ and subsequently as a 13 item short form.

The Patient Activation Measure (PAM®) is a tool for measuring the level of patient engagement in their healthcare. It is defined as having knowledge to manage one's condition and maintain functioning and prevent health declines; skills and behavioural repertoire to manage their condition, abilities to collaborate with their health providers, maintain their health functioning, and access appropriate and high-quality care. The PAM ${ }^{1} 13$-item scale enquires about people's beliefs, knowledge and confidence for engaging in health behaviours and assigns an activation score based on their responses to the 13-item scale. They also report that the PAM has good psychometric properties indicating that it can be used at the individual patient level to tailor interventions and assess changes.

The PAM $₫$-13 Kannada edition is composed of 13 items. It has five categories of highly disagreeable answer, disagree, agree, and strongly agree, not applicable scoring 1 to 5 respectively. The design of the instrument represents the four stages of activation in the processing complexity of the items: level 1 (patient believe that their role is important), Level 2 (patients lacks confidence and knowledge to take action of their health), Level 3 (patients indicating that patients began to take action for management of their health care) and Level 4 (patients had difficulty maintaining behaviours over time). Calculate a raw score: add up all of the responses to the 13 questions. For each "Strongly Disagree" response give the person a 1 , for each "Disagree" response give the person a 2, for each "Agree" response give the person a 3, for each "Strongly Agree" response give the person a 4

If there are no items with "not applicable" responses, use the total as the raw score. If there are items with a response of "not applicable" or a question left blank, these items are scored as "missing." In this case divide the point total by the number of items completed with a 14 response (exclude the missing) and multiply by 13 to get the raw score. For example, if two questions were answered with a "not applicable" response, your denominator would be 11 .

Second, convert the raw score into the activation score. If necessary, round off your raw score to the nearest whole number. Then, using the table below, simply find the raw score in the column marked "Raw Score," then read across to find the person's activation score. The raw score was transformed into natural logarithm according to Insignia Health Inc. guidelines to achieve a clearer representation of the relative distance between the scores. Therefore, these objects have been converted into a standardized metric of 0 100 (0- low activation and 100- maximum activation). The scores were calculated by summing up the raw scores and mapping up the sums into a scale of 0-100.

\section{Statistical Analysis}

The data was analysed using SPSS 24.0 for Mean, Median, Standard Deviation, Pearson's and Spearman's Correlation. Descriptive data were used to determine the mean and standard deviations. Stata 14 was used to analyse Internal consistency using Cronbach's Alpha, Inter-item, Inter-Rest, Inter-test correlation. The level of significance for analysis was set at $\leq 0.05$.

\section{RESULTS}

200 subjects were enrolled in the study according to eligibility criteria. Males accounted for $65 \%$ with a mean 
PAM $® 13$ of $55.69( \pm 11.67)$ and females accounted for $34.5 \%$ with a mean PAM®13 of $55.65( \pm 11.83)$ and p-value 0.984 which was not statistically significant.

Patients aged from 18 to 80 years with subjects $<45$ aged with mean PAM $® 13$ of 61.27 , age group of $45-64$ with mean PAM ${ }^{13}$ of 55.99 and age group $>65$ years with mean PAM $® 13$ of 54.72 with $p$ value of 0.260 which was with mean of $62.69( \pm 11.74)$ which was not statistically significant. With increasing age group, the level of activation significantly reduced. Pearson's correction was taken between age group with $r=-0.117$.

\begin{tabular}{|c|c|c|c|c|c|}
\hline \multicolumn{2}{|c|}{ Characteristic } & \multirow{3}{*}{$\begin{array}{c}\text { Total Number } \\
131 \\
69\end{array}$} & \multicolumn{3}{|c|}{ Percentage PAM@-13 Score P-Value } \\
\hline \multirow[b]{2}{*}{ Gender } & Male & & $65 \%$ & 55.69 & \multirow{2}{*}{0.984} \\
\hline & Female & & $34.5 \%$ & 55.65 & \\
\hline & $<45$ & 9 & $4.5 \%$ & 61.27 & \multirow{3}{*}{0.260} \\
\hline \multirow[t]{2}{*}{ Age group } & $45-65$ & 104 & $52 \%$ & 55.99 & \\
\hline & $>65$ & 87 & $43.5 \%$ & 54.72 & \\
\hline \multirow{2}{*}{ Education } & 12 & 51 & $25.5 \%$ & 50.11 & \multirow{2}{*}{$<0.001$} \\
\hline & $12+$ & 149 & $74.5 \%$ & 57.61 & \\
\hline
\end{tabular}

\begin{tabular}{|c|c|c|}
\hline Level of PAMß-13 & Frequency & Percentage \\
\hline Level 1 & 53 & 26.5 \\
\hline Level 2 & 37 & 18.5 \\
\hline Level 3 & 91 & 45.5 \\
\hline Level 4 & 17 & 8.5 \\
\hline
\end{tabular}

\begin{tabular}{|c|c|c|c|c|c|c|c|c|c|c|}
\hline 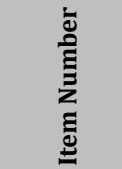 & $\sum^{0}$ & 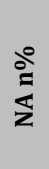 & $\begin{array}{l}\stackrel{0}{\Xi} \\
\text { ڤิ }\end{array}$ & $\stackrel{\ominus}{a}$ & 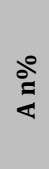 & 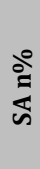 & 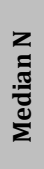 & $\begin{array}{l}\text { J } \\
\sum \\
\Sigma\end{array}$ & 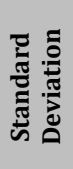 & 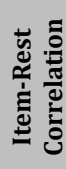 \\
\hline PAM13-1 & 0 & 0 & 2 & 38 & 131 & 29 & 3 & 2.94 & 0.61 & 0.43 \\
\hline PAM13-2 & 0 & 0 & 3 & 26 & 128 & 43 & 3 & 3.06 & 0.63 & 0.48 \\
\hline PAM13-3 & 0 & 2 & 2 & 19 & 119 & 58 & 3 & 3.18 & 0.63 & 0.47 \\
\hline PAM13-4 & 0 & 3 & 3 & 24 & 121 & 49 & 3 & 3.10 & 0.65 & 0.42 \\
\hline PAM13-5 & 0 & 10 & 22 & 66 & 76 & 26 & 3 & 2.56 & 0.86 & 0.44 \\
\hline PAM13-6 & 0 & 13 & 24 & 47 & 85 & 31 & 3 & 2.66 & 0.90 & 0.41 \\
\hline PAM13-7 & 0 & 11 & 6 & 41 & 117 & 24 & 3 & 2.85 & 0.67 & 0.33 \\
\hline PAM13-8 & 0 & 7 & 1 & 31 & 124 & 37 & 3 & 3.02 & 0.61 & 0.43 \\
\hline PAM13-9 & 0 & 9 & 4 & 24 & 111 & 52 & 3 & 3.10 & 0.68 & 0.41 \\
\hline PAM13-10 & 0 & 4 & 4 & 34 & 95 & 63 & 3 & 3.11 & 0.75 & 0.41 \\
\hline PAM13-11 & 0 & 3 & 5 & 30 & 115 & 47 & 3 & 3.04 & 0.70 & 0.52 \\
\hline PAM13-12 & 0 & 1 & 9 & 27 & 129 & 33 & 3 & 2.94 & 0.69 & 0.55 \\
\hline PAM13-13 & 0 & 6 & 13 & 43 & 92 & 46 & 3 & 2.88 & 0.84 & 0.54 \\
\hline \multicolumn{11}{|c|}{ Table 3. Description of the PAM $囚-13$, Kannada Version } \\
\hline 18 & & & & & & & & & & ee, SA \\
\hline
\end{tabular}

Educational level $(12+)$ accounted for $74.5 \%$ with mean of $57.61( \pm 11.38)$ which was statistically significant.

The frequency of scores seen in Level $1(n=53)$ i.e. $26.5 \%$ of patients may not believe that the patient role is important, Level $2(n=37) 18.5 \%$ i.e. of patients lacks confidence and knowledge to take action of their health, Level $3(n=91)$ i.e. $45 \%$ of patients indicating that patients began to take action for management of their health care and Level $4(\mathrm{~N}=17)$ i.e. 8 $\%$ of patients had difficulty maintaining behaviours over time

This table describes the psychometric properties of the PAM13 of the Kannada version with data quality (missing value), internal consistency, reliability, item-rest correlation, item- total correlation and average inter-item correction.

Percentage of missing value was 0 with "Not Applicable" (ranging between 0.5 to $6.5 \%$ ).The frequencies of the response categories in the study showed little use of the category "Strongly Disagree" and moderate use of "Disagree" and "Agree". The frequency of using the category, "Strongly Agree" was highest for all the items. The median score was 3 for all the 13 items. The mean scores ranged from 2.56 to
3.18. Standard deviation showed variability in all items, ranging from 0.61 to 0.90 .

A Cronbach's alpha equal to 0.84 for the sum scale was considered a very good level of internal consistency (0.6$0.88)$. The inter-rest correlation per item to the sum scale were ranged from 0.38 to 0.58 . The inter-item correlations ranged from 0.27 to 0.29 , with an Average inter item correlation equal to 0.28 .

\section{DISCUSSION}

The study was aimed at translating and validating the PAM $\AA$ -13 questionnaires into the Kannada language. This study evaluated the PAM ${ }^{\circledR}-13$ 's reliability, validity, and responsiveness in the Kannada language. Diabetes is one of the most prevailing chronic conditions and a major cause of morbidity, disability and mortality ${ }^{11}$ with an incidence of 31.7 million in India. It is more prevalent in southern India as compared to northern India. In a national urban survey, 12.4 $\%$ of total population accounts for Bangalore. ${ }^{11}$ High response rate was obtained with a low percentage of missing items (2 per cent), reducing the risk of bias.

In the present study, the level of internal consistency is 0.8357 which is less compared to Italian ${ }^{5}$ (0.88), German 4 (0.88), Danish ${ }^{7}$ (0.89) and Dutch ${ }^{8}(0.88)$ translations and is higher to Hebrew language (0.77). ${ }^{6}$ Internal consistency could be strengthened by rephrasing item 5 , it em 6 and item 7 of PAM $₫ 13$ which has the highest percentag e of missing values in the Kannada version. ${ }^{3}$ Internal consistency could be improved by rephrasing item 5 (I am confident that I can tell whether I need to go to the doctor or whether I can take care of a health problem myself), item 6 (I am confident that I can tell a doctor concerns I have even when he or she does not ask), item 7 (I am confident that I can follow through on medical treatments I may need to do at home) which has the highest percentage of missing values. A high inappropriate level of "non-applicable" scores and a relatively low item-rest correlation is mentioned in table no 4.

The frequency of the responses in the study shows, minimal use of the "strongly disagree" and adequate use of "disagree" and "agree". The frequency of using "strongly agree" was highest for all the items. Due to low responsiveness, the studies of Dutch ${ }^{8}$ and Hebrew 6 did not publish the data of frequency.

The mean PAM $₫-13$ score for the Kannada version was 55.6812 which was lower than the average of Italian 66.54, German 68.3 5, Danish 64.2, Hebrew 70.7.6. This may be attributed to the various sample sizes, various sample characteristics related to educational level, therapist and patient relationship, health care facilities and their diagnostic heterogeneity.

The mean age of the German (67) ${ }^{4}$ was higher than the present study mean age (62.9) and lower than Danish (62) ${ }^{7}$ and Dutch $(59)^{8}$ patient, it could be due to respondents have been diagnosed recently with diabetes ${ }^{14,15}$.

In current study, age group above 65 years reported lower PAM® -13 scores. They are exposed to increased variety of personal and social conditions as patients age that challenge their sense of control and independence. This leads to low efficiency in managing their health. This result is in 
accordance with the previous study.7,16 As patients aged, they are exposed to increasing variety of personal and social conditions that challenge their sense of control and independence. The tendency for individuals with low perceived self-efficiency to engage in health-promoting behaviours becomes stronger in older adults mainly because of physical decline which is considered unalterable part of aging 17 .

The reason for lower PAM $₫-13$ in older individuals could be due to lower health literacy, reluctance to seek help for their complaints and obtaining information during medical interviews and participate less in their medical consultations and often have multiple health problems ${ }^{18}$. Therefore in the study PAM® - 13 score was lower in age group of above 65 years of age.

In the present study, there was larger enrolment of male population than female. Hence, there was no uniform distribution between genders for the PAM ${ }^{\circledR}$ which was not statistically significant.

When the educational level was compared with the PAM®-13 score, it was statistically significant as higher education and income tend to have a strong positive association with the patient activation score. There is easy accessibility of health care services which increases knowledge about one's own health ${ }^{19}$.

In India with a wide range of chronic conditions, there is different care prescription ranging from routine examination, regular medication and follow up due to increasing knowledge about the health care due to easy accessibility of health care services in Indian set up there is low cost of health care services which leads to high activation of selfmanagement skills. When chronicity of disease was correlated with PAM-13 it showed to have statistically significant weak negative Correlation.

In the Insignia Health Inc.'s measure of patient activation (PAM $®-13$ ), each activation level is indicated by following descriptions:

- Level 1 - the patient might not feel that his role is important.

- Level 2 - the patient does not take action due to lack of confidence and knowledge.

- Level 3 - the patient begins to take action.

- Level 4 - the patient has difficulty in maintaining their behaviour. ${ }^{20,21,22}$

In the present study, cumulative score of Level $1(n=53)$ i.e. $26.5 \%$ of patients may not believe that the patient role is important, Level $2(n=37) 18.5 \%$ i.e. of patients lacks confidence and knowledge to take action of their health, Level $3(n=91)$ i.e. $45 \%$ of patients indicating that patients began to take action for management of their health care and Level 4 $(\mathrm{N}=17)$ i.e. $8 \%$ of patients had difficulty maintaining behaviours over time.

\section{CONCLUSIONS}

PAM $®-13$ in Kannada language has demonstrated to be a reliable and valid measure, as it allows adaptation of the instrument in Kannada language and cross cultural adaptation was emphasized and achieved. This translation of Kannada language will lead to efficient communication with the patients and to tailor healthcare message and selfmanagement goals. This translated version of PAM® - 13 in Kannada language can be used as an outcome measure to check for the awareness of patient's health status in diabetic population and plan exercise prescription for patients with diabetes. As mentioned in the previous studies, this translated version can also be used for chronic illnesses in the State of Karnataka, India. This will lead to greater improvement in clinical status, enhances the medication regime and will lead to shorter hospitalization.

\section{Future Recommendation}

This translated version of PAM $® 13$ in Kannada language can be used as an outcome measure to check for the awareness of patient's health status in diabetic population and to plan exercise prescription for patients with diabetes. As mentioned in the previous studies, this translated version can also be used for chronic illnesses in the State of Karnataka, India.

Financial or Other Competing Interests: None.

\section{REFERENCES}

[1] Anderson G, Horvath J. The growing burden of chronic disease in America. Public Health Rep 2004;119(3):26370.

[2] Hibbard JH, Mahoney ER, Stockard J, et al. Development and testing of a short form of the patient activation measure. Health Serv Res 2005;40(6 Pt 1):1918-30.

[3] Hibbard JH, Stockard J, Mahoney ER, et al. Development of the patient activation measure (PAM): conceptualizing and measuring activation in patients and consumers. Health Serv Res 2004;39(4 Pt 1):1005-26.

[4] Brenk-Franz K, Hibbard JH, Herrmann WJ, et al. Validation of the German version of the patient activation measure 13 (PAM13-D) in an international multicentre study of primary care patients. PLoS One 2013;8(9):e74786.

[5] Graffigna G, Barello S, Bonanomi A, et al. Measuring patient activation in Italy: translation, adaptation and validation of the Italian version of the patient activation measure 13 (PAM13-I). BMC Med Inform Decis Mak 2015;15:109.

[6] Magnezi R, Glasser S. Psychometric properties of the hebrew translation of the patient activation measure (PAM-13). PLoS One 2014;9(11):e113391.

[7] Maindal HT, Sokolowski I, Vedsted P. Translation, adaptation and validation of the American short form Patient Activation Measure (PAM13) in a Danish version. BMC Public Health 2009;9:209.

[8] Rademakers J, Nijman J, van der Hoek L, et al. Measuring patient activation in the Netherlands: translation and validation of the American short form Patient Activation Measure (PAM13). BMC Public Health 2012;12:577.

[9] Greene J, Hibbard JH. Why does patient activation matter? An examination of the relationships between patient activation and health-related outcomes. J Gen Intern Med 2012;27(5):520-6. 
[10] Remmers C, Hibbard J, Mosen DM, et al. Is patient activation associated with future health outcomes and healthcare utilization among patients with diabetes? J Ambul Care Manage 2009;32(4):320-7.

[11] Kaveeshwar SA, Cornwall J. The current state of diabetes mellitus in India. Australas Med J 2014;7(1):45-8.

[12] CDC - NCHS - National Centre for Health Statistics 2020. https://www.cdc.gov/nchs/index.html

[13] WHO. Health systems strengthening glossary. World Health Organization 2013. https://www.who.int/healthsystems/hss_glosary/en/in dex5.html

[14] Renders CM, Valk GD, Griffin SJ, et al. Interventions to improve the management of diabetes in primary care, outpatient, and community settings: a systematic review. Diabetes Care 2001;24:1821-33.

[15] Wagner EH, Austin BT, Von Korff M. Improving outcomes in chronic illness. Managed Care Q 1996;4(2):12-25.

[16] Lorig K, Holman H. Self-management education: history, definition, outcomes, and mechanisms. Ann Behav Med 2001;26(1):1-7.
[17] Mead H, Andres E, Ramos C, et al. Barriers to effective self-management in cardiac patients: the patient's experience. Patient Education and Counselling 2010;79(1):69-76.

[18] Mayberry R, Willock RJ, Boone L, et al. A high level of patient activation is observed but unrelated to glycemic control among adults with type 2 diabetes. Diabetes Spectr 2010;23(3):171-6.

[19] Hendriks M, Rademakers J. Relationships between patient activation, disease- specific knowledge and health outcomes among people with diabete; a survey study. BMC Health Serv Res 2014;14:393.

[20] Shah VO, Carroll C, Mals R, et al. A home-based educational intervention improves patient activation measures and diabetes health indicators among Zuni Indians. PLoS One 2015;10(5):e012520.

[21] Insignia Health: Patient activation measure (PAM) $13^{\text {тM }}$ License Materials (C) LLC 2013.

[22] World Health Organization. Process of translation and adaptation of instruments. 2010. http://who.int/substance_abuse/research_tools/transl ation/en/. 